\title{
Newly Diagnosed Multiple Myeloma: How Many Drugs Are Enough?
}

\author{
Presented by Shaji K. Kumar, MD
}

\begin{abstract}
The treatment of multiple myeloma ( $\mathrm{MM}$ ) has evolved over the past decade, yet it remains a chronic disease. Several trials of 4-drug induction regimens have resulted in deepening of disease response. With the emergence of multidrug regimens, questions have arisen regarding the role of autologous stem cell transplant (ASCT) in MM therapy and available treatment options after ASCT. Clinicians have also continued to improve the efficacy of maintenance therapies. In transplant-ineligible patients, the phases of treatment are less distinct; however, several regimens have demonstrated efficacy in this clinical setting. Future research should focus on individualizing treatment approaches.
\end{abstract}

J Natl Compr Canc Netw 2021;19(11.5):1347-1350 doi: 10.6004/jnccn.2021.5104

Over the past decade, treatment for patients with newly diagnosed multiple myeloma (MM) has evolved. At the NCCN 2021 Virtual Congress: Hematologic Malignancies, Shaji K. Kumar, MD, Mark and Judy Mullins Professor of Hematologic Malignancies, Mayo Clinic Cancer Center, discussed the current treatment options and reviewed emerging therapies.

\section{Treatment Paradigm}

On patient presentation, according to Dr. Kumar, it is critical to accurately diagnose active MM by distinguishing it from precursor plasma cell disorders such as MGUS and smoldering myeloma. After diagnosis, the next step in treatment is risk stratification.

Initial treatment for MM should be tailored to achieve the maximum possible depth of response that can be sustained for the longest period of time. However, in the majority of patients, relapse is likely to occur. Dr. Kumar noted that multiple lines of therapy may be required to achieve sustained disease control over time. Transplant-eligible patients are usually administered induction therapy, consolidation therapy with autologous stem cell transplant (ASCT), and maintenance therapy with one or more drugs. For those who are transplant-ineligible, the phases of treatment are not as clear-patients are often continued on the drugs administered at diagnosis (Figure 1).

"Both of these approaches should allow us to achieve sustained disease control," he explained. "With most currently used initial therapies, this often lasts from 3 to 5 years."

\section{Current Standard of Care for Newly Diagnosed MM}

The current standard of care for newly diagnosed MM is the combination of bortezomib/lenalidomide/dexamethasone, which was validated in the SWOG S0777 study. ${ }^{1}$ In this trial, patients were randomly assigned to receive lenalidomide + dexamethasone \pm bortezomib. Based on the results of this study, the addition of bortezomib improved progression-free survival (PFS) and overall survival (OS) in this patient population. However, according to Dr. Kumar, the incidence rates of gastrointestinal and neurologic toxicities appeared to increase with this drug combination.

\section{Improving MM Induction Therapy}

"Over the past decade, we have continued to improve upon the initial therapy for MM," Dr. Kumar commented. "One such approach was to replace bortezomib with another proteasome inhibitor [that does not seem to be] associated with peripheral neuropathy."

In the multicenter phase III ENDURANCE trial, ${ }^{2}$ patients were randomly assigned to receive lenalidomide + dexamethasone in combination with bortezomib or carfilzomib. Replacing bortezomib with carfilzomib did not seem to improve PFS $(P=.742)$; however, patients treated with carfilzomib appeared to experience a deeper response. According to Dr. Kumar, the lack of improvement in PFS may be due to the unique toxicity profile of carfilzomib.

Another approach to improve treatment outcomes was the addition of the monoclonal antibody daratumumab to bortezomib + thalidomide and dexamethasone. In the phase III CASSIOPEIA trial, ${ }^{3}$ transplant-eligible patients with newly diagnosed MM treated with this combination appeared to experience an improved depth of response compared with those who did not receive daratumumab; this benefit was observed in all subgroups, except in those with high-risk disease. Dr. Kumar 


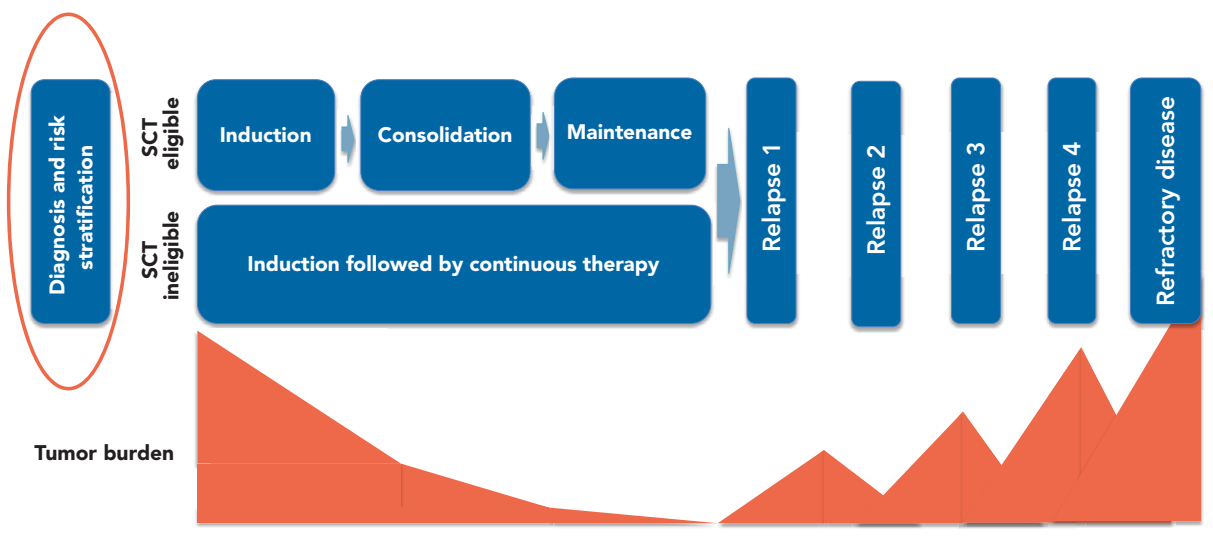

Figure 1. Myeloma treatment paradigm.

Abbreviation: $\mathrm{SCT}$, stem cell transplant.

noted that the addition of daratumumab improved PFS (hazard ratio, 0.58) and OS (hazard ratio, 0.54).

Given that combination thalidomide is not often used in the United States, the phase II GRIFFIN trial was conducted to evaluate daratumumab + bortezomib/ lenalidomide/dexamethasone in a similar patient population. ${ }^{4}$ The findings from the study were comparable to those reported in the CASSIOPEIA trial: the response rates and depths were greater in patients treated with daratumumab than in those who did not receive it. In addition, there was a trend toward improved PFS with the addition of daratumumab.

Additionally, according to Dr. Kumar, the results of a phase II trial of the proteasome inhibitor ixazomib + dexamethasone/lenalidomide/daratumumab revealed a deepening of response with continued therapy. ${ }^{5}$ This 4-drug regimen also appeared to have a manageable overall toxicity profile. "One of the advantages of 4-drug regimens is that now we can achieve deeper responses as part of induction therapy than what we have seen with 3-drug combinations," he explained. "With the more effective 4 -drug regimens..., the hope is that we can now limit the duration of therapy."

According to Dr. Kumar, investigators of the phase II MASTER trial sought to limit the duration of combination therapy with daratumumab/carfilzomib/lenalidomide/ dexamethasone based on depth of response. ${ }^{6}$ By the end of consolidation therapy, most patients had achieved measurable residual disease (MRD) negativity.

\section{MRD Negativity and High-Risk MM}

"[The results of the MASTER trial] speak to the potential of MRD negativity as a clinically actionable tool for directing therapy," Dr. Kumar remarked. This seems to be especially relevant in the setting of high-risk MM. If these patients achieve sustained MRD negativity, they may experience improved survival outcomes.
Several trials with multidrug combinations have been initiated to assess this relationship. In the OPTIMUM trial, ${ }^{7}$ a high proportion of patients who received the 5 -drug induction regimen of daratumumab + cyclophosphamide/bortezomib/lenalidomide/dexamethasone achieved MRD negativity. Dr. Kumar noted that these early results may translate into better long-term outcomes.

\section{Do We Still Need ASCT?}

According to Dr. Kumar, with the emergence of multidrug regimens, questions have arisen regarding the role of ASCT in the treatment of MM. In the phase III IFM/ DFCI 2009 trial, ${ }^{8}$ patients were randomly assigned to receive lenalidomide + bortezomib and dexamethasone \pm ASCT. Those who underwent ASCT experienced a longer PFS duration than those who did not.

"ASCT is still important as part of treatment for MM," he commented. "Whether we use it as part of either the initial package or the second-line of therapy, we derive a similar degree of benefit in terms of OS for these patients."

Data supporting ASCT in this clinical setting also come from a trial of patients randomly assigned to receive a carfilzomib-based regimen \pm ASCT. After the first randomization, patients who received carfilzomib + lenalidomide and dexamethasone followed by ASCT derived the greatest PFS benefit in all risk cohorts. ${ }^{9}$ "Clearly, ASCT plays a major role irrespective of the underlying risk stratification,” Dr. Kumar noted.

\section{What Should Be Done After ASCT?}

According to Dr. Kumar, there are 3 treatment options after ASCT: consolidation therapy with tandem ASCT, nontransplant consolidation therapy, or maintenance therapy. In the STaMINA trial, ${ }^{10}$ after undergoing ASCT, patients were randomly assigned to either maintenance therapy with 
lenalidomide, consolidation therapy with bortezomib + lenalidomide and dexamethasone, or a tandem transplant. Study results showed that the method of treatment administered after ASCT did not appear to affect PFS outcomes.

In contrast, the EMN02/HO95 trial demonstrated that patients with high-risk cytogenetics may benefit from tandem ASCT. ${ }^{11}$ These findings have also been shown in retrospective studies of tandem ASCT versus a single transplant. "There is probably a role for tandem ASCT for patients with high-risk disease,” Dr. Kumar explained. “[It] certainly should be part of the treatment discussion with the patient."

\section{Approaches to Maintenance Therapy}

"In the majority of the patients in our practice who are undergoing [bortezomib + lenalidomide and dexamethasone] induction therapy and a single ASCT, it is reasonable to transition to a maintenance therapy," Dr. Kumar commented. "The question is: What do we use for maintenance therapy?"

A meta-analysis of 3 phase III trials revealed that administering lenalidomide maintenance therapy after ASCT improved PFS and OS outcomes. ${ }^{12}$ Additionally, in the phase III TOURMALINE-MM3 trial, maintenance therapy with ixazomib significantly improved PFS outcomes compared with placebo $(P=.002) .{ }^{13}$ The CASSIOPEIA trial demonstrated that patients who underwent initial and/or maintenance therapy with daratumumab experienced improved outcomes. ${ }^{3}$

Maintenance therapy with lenalidomide alone may be insufficient in patients with high-risk disease, according to Dr. Kumar. Data from a randomized trial of lenalidomide \pm carfilzomib support the use of lenalidomide plus a proteasome inhibitor in this clinical setting. ${ }^{9}$

\section{Treatment of Transplant-Ineligible Patients}

A less-intense induction therapy regimen of lenalidomide/bortezomib/dexamethasone (VRd-lite) has been studied in the transplant-ineligible population. Patients treated with this regimen had a median duration PFS of approximately 3 years. ${ }^{14}$

The MAIA trial was designed to improve on that regimen by adding daratumumab to lenalidomide + dexamethasone. Patients who received daratumumab experienced a significantly improved overall response rate and depth of response, which also translated into a PFS $(P<.0001)$ and OS ( $P=.0013$ ) benefit. ${ }^{15}$ According to Dr. Kumar, daratumumab + lenalidomide and dexamethasone has become a standard regimen for transplant-ineligible patients with newly diagnosed MM.

The combination of daratumumab + bortezomib/ melphalan/prednisone is commonly administered in Europe, and has become another alternate approach for the treatment transplant-ineligible patients. In the ALCYONE trial, ${ }^{16}$ patients who received this regimen experienced deeper responses and better PFS and OS outcomes than those who did not receive daratumumab.

The TOURMALINE-MM2 trial of lenalidomide + dexamethasone in combination with ixazomib or a placebo failed to meet the prespecified threshold for improved PFS. ${ }^{17}$ However, the median PFS duration was found to be similar to that of the standard and less-intense lenalidomide/bortezomib/dexamethasone regimens.

\section{Conclusions}

Patients with MM treated with 4-drug induction regimens experience deeper responses, which may translate to better outcomes. The initial treatment strategy may have significant implications on the long-term outcomes of this population, according to Dr. Kumar. "I think the key is to deliver the most effective 'package' of treatment that we can give these patients while... [keeping the] toxicity-related issues at the minimum and improving the quality of life."

Dr. Kumar highlighted the importance of the riskstratified treatment approach. After patients with highrisk disease have achieved MRD negativity, they should be administered a multidrug maintenance therapy.

"Clearly, there is a lot more that needs to be done in terms of individualizing the treatment approach," he concluded. "With [the] more therapies we have on hand, we will continue to improve upon that."

Disclosures: Dr. Kumar has disclosed receiving grant/research support and serving as a scientific advisor for AbbVie, Inc., Amgen Inc., Bristol-Myers Squibb Company, GlaxoSmithKline, Janssen Pharmaceutica Products, LP, Karyopharm Therapeutics, Regeneron Pharmaceuticals, Inc., Roche Laboratories, Inc. Sanofi-aventis US, and Takeda Pharmaceuticals North America, Inc; and receiving honoraria from Oncopeptides and BeiGene.

Correspondence: Shaji K. Kumar, MD, Division of Hematology, Mayo Clinic Cancer Center, 200 First Street SW, Rochester, MN 55905.

Email: kumar.shaji@mayo.edu

\section{References}

1. Durie BGM, Hoering A, Abidi MH, et al. Bortezomib with lenalidomide and dexamethasone versus lenalidomide and dexamethasone alone in patients with newly diagnosed myeloma without intent for immediate autologous stem-cell transplant (SWOG S0777): a randomised, openlabel, phase 3 trial. Lancet 2017:389:519-527.

2. Kumar SK, Jacobus SJ, Cohen AD, et al. Carfilzomib or bortezomib in combination with lenalidomide and dexamethasone for patients with newly diagnosed multiple myeloma without intention for immediate

autologous stem-cell transplantation (ENDURANCE): a multicentre, open-label, phase 3, randomised, controlled trial. Lancet Oncol 2020;21: 1317-1330.

3. Moreau P, Attal M, Hulin C, et al. Bortezomib, thalidomide, and dexamethasone with or without daratumumab before and after autologous stem-cell transplantation for newly diagnosed multiple myeloma (CASSIOPEIA): a randomised, open-label, phase 3 study. Lancet 2019. 394:29-38 
4. Voorhees PM, Kaufman JL, Laubach JP, et al. Depth of response to daratumumab, lenalidomide, bortezomib, and dexamethasone (RVd) improves over time in patients with transplant-eligible newly diagnosed multiple myeloma: Griffin study update [abstract]. Blood 2019;134(Suppl 1):Abstract 691

5. Kapoor P, Gertz MA, Laplant B, et al. Phase 2 trial of daratumumab, ixazomib, lenalidomide and modified dose dexamethasone in patients with newly diagnosed multiple myeloma [abstract]. Blood 2019;134(Suppl 1): Abstract 864.

6. Costa LJ, Chhabra S, Godby KN, et al. Daratumumab, carfilzomib, lenalidomide and dexamethasone (Dara-KRd) induction, autologous transplantation and post-transplant, response-adapted, measurable residual disease (MRD)-based Dara-KRd consolidation in patients with newly diagnosed multiple myeloma [abstract]. Blood 2019:134(Suppl 1): Abstract 860

7. Kaiser MF, Hall A, Walker K, et al. Depth of response and minimal residual disease status in ultra high-risk multiple myeloma and plasma cell leukemia treated with daratumumab, bortezomib, lenalidomide, cyclophosphamide and dexamethasone (Dara-CVRd): results of the UK Optimum/MUKnine trial [abstract]. J Clin Oncol 2021;39(Suppl 15): Abstract 8001

8. Attal M, Lauwers-Cances V, Hulin C, et al. Autologous transplantation for multiple myeloma in the era of new drugs: a phase III study of the Intergroupe Francophone Du Myelome (IFM/DFCl 2009 trial) [abstract]. Blood 2015;126:Abstract 391.

9. Gay F, Mina R, Rota-Scalabrini D, et al. Carfilzomib-based induction/consolidation with or without autologous transplant (ASCT) followed by lenalidomide (R) or carfilzomib-lenalidomide (KR) maintenance: efficacy in high-risk patients [abstract]. J Clin Oncol 2021;39(Suppl):Abstract 8002

10. Hari P, Pasquini MC, Stadtmauer EA, et al. Long-term follow-up of BMT CTN 0702 (STaMINA) of postautologous hematopoietic cell transplantation (AutoHCT) strategies in the upfront treatment of multiple myeloma (MM) [abstract]. J Clin Oncol 2020;38(Suppl):Abstract 8506.

11. Cavo M, Gay FM, Patriarca F, et al. Double autologous stem cell transplantation significantly prolongs progression-free survival and overall survival in comparison with single autotransplantation in newly diagnosed multiple myeloma: an analysis of phase 3 EMN02/HO95 study [abstract]. Blood 2017;130(Suppl 1):Abstract 401

12. McCarthy $\mathrm{PL}$, Holstein $\mathrm{SA}$, Petrucci $\mathrm{MT}$, et al. Lenalidomide maintenance after autologous stem-cell transplantation in newly diagnosed multiple myeloma: a meta-analysis. J Clin Oncol 2017;35:3279-3289.

13. Dimopoulos MA, Gay F, Schjesvold F, et al. Oral ixazomib maintenance following autologous stem cell transplantation (TOURMALINE-MM3): a double-blind, randomised, placebo-controlled phase 3 trial. Lancet 2019, 393:253-264.

14. O'Donnell EK, Laubach JP, Yee AJ, et al. A phase 2 study of modified lenalidomide, bortezomib and dexamethasone in transplant-ineligible multiple myeloma. Br J Haematol 2018;182:222-230.

15. Facon T, Kumar SK, Plesner T, et al. Overall survival results with daratumumab, lenalidomide, and dexamethasone versus lenalidomide and dexamethasone in transplant-ineligible newly diagnosed multiple myeloma: phase 3 MAIA study [abstract]. Presented at the European Hematology Association (EHA) Congress 2021; June 9-17, 2021. Abstract LB1901.

16. Mateos MV, Cavo M, Bladé J, et al. Daratumumab plus bortezomib, melphalan, and prednisone versus bortezomib, melphalan, and prednisone in patients with transplant-ineligible newly diagnosed multiple myeloma: overall survival in ALCYONE [abstract]. Blood 2019;134(Suppl 1): Abstract 859

17. Facon $\mathrm{T}$, Venner $\mathrm{CP}$, Bahlis NJ, et al. Oral ixazomib, lenalidomide, and dexamethasone for transplant-ineligible patients with newly diagnosed multiple myeloma. Blood 2021;137:3616-3628. 